\title{
The Applied Research of Video Image Processing Technology in Athletics Programs
}

\author{
WenJin $^{1}$ a \\ 1Sports Department of Zhengzhou Normal University, Zhengzhou, 450000,China. \\ aemail:15203713869@163.com
}

Keywords: motion video image; capture technology; applied research; athletics

\begin{abstract}
In this paper, it adopts the document literature and comprehensive analysis methods and it studies the use of video capture images in athletics programs. Then it draws the appropriate conclusions, so that the athletic coaches will use and guide the athletes a reasonable training.
\end{abstract}

\section{Foreword}

People use a variety of tools and means in different forms to obtain video images from the objective world observations. it can be directly or indirectly act on the human eyes, and produce visual perception entity, which is the static or dynamic images video human eyes observed. This is a broader definition of the image. Scientific research and statistics show that about $75 \%$ information obtained from the outside world is from the visual system, which is namely obtained from the image. Sports biomechanics and athletic training, especially the latter, in terms of the movement technical level diagnosis and evaluation for the athletes, frequently uses video images to study. Through the two-dimensional or three-dimensional analysis of motion technique video images, obtain images or data information, the athletes technically showed. And through scientific analysis and evaluation, assess the level of technique training, and it is built on the basis of video images. So the image processing techniques can intuitively make the technical action monitoring and evaluation for the athletes, and stimulate athletes' learning, improve athletic skills interest. Meanwhile the motion technique video is conducted without interference for athletes, directly gets the real technical information of competition and training site. And this can best meet the requirements of sports training and the real situation of the competition.

\section{Brief Introduction on Image Processing Technology}

In a broad sense, image technology is a general term for a variety of image-related technologies. At present, people mainly study the digital images, which the main application is computer graphics technology. This includes a series of work completed by using the computers and other electronic devices, such as the image acquisition, image coding, storage and transport, image synthesis and generation, image display and output, image transformation, enhancement, restoration (recovery) and reconstruction, image segmentation, object detection, expression and description, feature extraction and measurement, correction of image sequence, 3D (three-dimensional) scene reconstruction and restoration, establishment, index, and extraction of the image database, image classification, representation and recognition, image models establishment and matching, images and scenes interpretation and understanding, as well as the judgment and decision, behavior planning and so on. 1980s and 1990s, the image processing technology can not only be used in 2D (two-dimensional) space outside to make processing and application[1], but also the application and development of the 3D space has also been booming. Image technology has been related to human life and social development in all aspects. In the 21st century, the image processing technology will be further developed and applied. 
The development of image technology and the emergence of new image processing theory, methods, algorithms, tools, equipment, etc., generated the image engineering systems. Image Engineering can be divided into three levels: namely, image processing, image analysis and image understanding.

We do not need to have a very deep understanding on image processing technology, in sport scientific research, we are primarily used the hardware and software systems related with image processing technology[2].

\section{The Development of Several Image Acquisition and Processing New Technologies, As Well As Its Application in the Field of Sport Scientific Research}

2.1 DV camcorder improves the accuracy of the imaging, and the widespread use.

When DV camera is compared with the ordinary camcorder, which the latter has the use of VHS or S-VHS book analog signal, the horizontal resolution of the camera is about 500 lines. The accuracy is much higher than the AV camera image. And the DV camcorder is easy to carry, almost all DV camcorders are connected with LCD display. It's used for the fast feedback of live video in track and field training site[3].

2.2 Efficient transmission of IEEE1394 data stream

IEEE1394 is also known as Firewire (FireWire), which is a high-speed serial bus. Sony is the first company to add I.LINK terminal to portable DV camera, which meets the IEEE1394 standard. And this case makes the image data transfer rate greatly improved. As long as the hardware standard is under suitable condition, you can use IEE1394 interface, transfer images without distortion, and it's even better than the output results of using AV video.

In recent years, since other various brands of portable DV cameras have also added the I.LINK terminal, it makes I.LINK terminals become the indispensable input and output interface for digital imaging and computer connection. This also makes the DV camcorder almost completely replace the use of iVdos8, iVdoe Hi8, S-VHS and VHS-C and other analog signals cameras[4].

2.3 Mobile portable video acquisition and image processing system

Mobile portable video acquisition and image processing system is composed by an I.LINK terminal interface laptop, DV cameras and professional video analysis and image processing software. It uses the digital cameras and the laptop computer with IEEE1394 interface to transmit video images and get image acquisition. After this, the motion technology video can get further editing and processing through the special image processing software system, such as the continuous technical picture production, video overlap, the multimedia technological graphics text mixed and so on.

This new development makes image processing and analysis can be out of the laboratory and enter into practice technical training monitoring. This is particularly important for technical sports training and sports biomechanics in terms of tactical diagnosis.

\section{The Main Applied Forms of Video Image Processing Technology in Athletics Programs}

Video image processing technology has changed the previous feedback forms based on data and curves in technical training reports. Its motion video image, two-dimensional or three-dimensional dynamic pictures, continuous motion pictures, mixed data and curves and many other kinds of images and data feedback forms, make the image feedback more intuitive, practical and scientific.

3.1 The application on the motion technology image

3.1.1 Generally continuous motion technical pictures: Adopt the motion technical pictures of continuous acquisition at critical period, and this is a form of many researchers often used over the years.

3.1.2 Extract the synthetic image, from the critical period of continuous action technique: In the same context, extract the continuous multiple technical actions at the critical period of athletes, into one picture, and then get a complete technical synthetic picture.

3.1.3 The double-image comparison on motion technology image: it includes upper \& lower 
double-image or left \& right double-image comparison, in terms of comparing the technical movements of different players or the same player in different competitions or training[5].

3.1.4 The multi-image comparison on motion technology image

3.1.5 The synthesis picture of the technical image, key parameters and data curve: By appropriate computer image processing software or a dedicated image editing software, make the organic combination of motion technology image, key technical parameters and the variation curve of parameters. Then we can get the technical synthesis image, which is complete, multi-aspect, reflecting motion technical situations.

3.1.6 The three-dimensional motion technology simulation image: Through a three-dimensional analysis of motion technology image, you can simulate the three-dimensional motion technology simulation image, which can produce motion technology simulation image from different angles.

3.1.7 The identification and movement trajectories of the motion technical key points: When motion technology makes two or three dimensional analysis, we need to identify the key point of joints movement to show the changes of it in the form of track curves. And this can better reflect the motion technical situation.

3.1.8 The background processing of the motion technology image: According to the need, you can use specialized image processing technology software, removal, add and change the background of the moving subjects. That is, it can be removed from the background in accordance with the color of the specific picture subjects and the contour feature, etc. Then it can be used for further processing by other techniques.

3.2 The application of the video image technology

3.2.1 The short persistence of the interval venue at key period: Make the collection of technical action from the interval fields, then play it and keep the key phase temporarily in the same screen, and make continuous technological action video. It can be very easy and intuitive to observe the continuous variation features of the motion technological action.

3.2.2 Video superposition: This technique involves direct superposition of the still background under the same venue; the superposition under the same venue after the elimination of sports background; direct superposition of different venues under static background; the superposition under the different venue after the elimination of static (or motion) background; direct superposition of scanned image; the superposition after the elimination of scanned image.

32.3 multiple comparisons on video image: the superimposed comparison of a fixed motion period on video image

Split screen comparison of video image at regular motion period; superposed comparison of video image at a regular point; split screen comparison of multi-angle shot video image; split screen superposed comparison of multi-angle shot video image; superposed comparison of video image at key movement phase (or key frames). Of course, you can make the comparison on the same technology motion of the multiple digital video among two, three or many persons.

3.2.4 Arbitrarily slow play \& output of the video image

3.2.5 Multi-aspect enlargement process of video image: You can make enlargement demonstration on the key parts of the motion subjects in a same video image. And you can make those enlarged parts display in a same screen and reinforce the highlighting role of the main aspects in the study. This can facilitate the clearer observation and analysis.

3.2.6 The synchronous display on video files simulation image, charts and curves: In the same picture, it can simultaneously display the three-dimensional video image, the simulation of three-dimensional motion image, mutative charts and parametric curves of the key parameters. And this can make the video image contain more useful information content, which can comprehensively feedback on the qualitative and quantitative analysis of motion technology.

3.2.7 The production of technical action video VCD, DVD and other formats.

3.2.8 The calculation processing of key parameters video image: For video images, we can use a simple coordinate mark, scale measurement and angle measurement, etc., to calculate the time-varying key point distance, average speed and angle parameter data, and then it can facilitate technical characteristics of the motion for quantitative analysis. 
3.2.9 The manufacture on motion technology rhythm sound and video image of the elite athletes: For the run-up rhythm stability of jumping project, it has been a problem that the coaches and researchers are trying to solve. Through the application of video image technology, we can intensify the cadence rhythm for individual athletes at the best jump time, and then it can produce a best jump time the audio and video image file. This can help athletes to learn and master the best run-paced rhythm in visual perception.

3.2.10 Multimedia production and graphic mixed output system: use multimedia technology to produce video images, simulation image, motion trajectory or curve, text, stick figure and trajectory, dynamic data tables mixed and other graphics mixed output form.

\section{References}

[1] Zhou Xiaodong; Wu Chunxia; Zhou Xinglong, The development and application on training monitoring and performance evaluation systems [C], China Institute of Sports Science, the Eighth National Sports Science Conference Abstracts (a), 2007.

[2] SIMI Realit y M otion Sys tems GmbH. SIMI M ot ion UserManual[ CP/ DK] . 2002.

[3] Zhou Xinglong, Zhao Fang, Sequence motion image acquisition and the development of video analysis system [C], the Tenth National Sports Biomechanics Academic Exchange Conference, 2002.

[4] LiuXiao-Wei. Image processing technology [ M ] . BeiJing: QingHua University Press, 2004.

[5] SIMI Realit y M otion Sys tems GmbH. SIMI M ot ion UserManual[ CP/ DK] . 2002. 